\title{
Using Automated Learning Devices for Monkeys (ALDM) to study social networks
}

\author{
Nicolas Claidière $^{1}$ - Julie Gullstrand ${ }^{1} \cdot$ Aurélien Latouche $^{2}$ • Joël Fagot ${ }^{1}$
}

Published online: 16 December 2015

(C) Psychonomic Society, Inc. 2015

\begin{abstract}
Social network analysis has become a prominent tool to study animal social life, and there is an increasing need to develop new systems to collect social information automatically, systematically, and reliably. Here we explore the use of a freely accessible Automated Learning Device for Monkeys (ALDM) to collect such social information on a group of 22 captive baboons (Papio papio). We compared the social network obtained from the co-presence of the baboons in ten ALDM testing booths to the social network obtained through standard behavioral observation techniques. The results show that the co-presence network accurately reflects the social organization of the group, and also indicate under which conditions the co-presence network is most informative. In particular, the best correlation between the two networks was obtained with a minimum of 40 days of computer records and for individuals with at least 500 records per day. We also show through random permutation tests that the observed correlations go beyond what would be observed by simple synchronous activity, to reflect a preferential choice of closely located testing booths. The use of automatized cognitive testing therefore presents a new way of obtaining a large and regular amount of social information that is necessary to develop
\end{abstract}

Electronic supplementary material The online version of this article (doi:10.3758/s13428-015-0686-9) contains supplementary material, which is available to authorized users.

Nicolas Claidière

nicolas.claidiere@normalesup.org

1 Laboratoire de Psychologie Cognitive UMR 7290, Aix Marseille Université and Centre Nationale de la Recherche Scientifique, Marseille 13331, France

2 EA 4629, Conservatoire National des Arts et Métiers, Paris, France social network analysis. It also opens the possibility of studying dynamic changes in network structure with time and in relation to the cognitive performance of individuals.

Keywords Animal behaviour $\cdot$ Baboon $\cdot$ Computerised testing $\cdot$ Social cognition

Social network analysis (SNA) has become a prominent tool to study the social life of animals in general (Croft, James, \& Krause, 2008; Krause, Lusseau, \& James, 2009; Kurvers, Krause, Croft, Wilson, \& Wolf, 2014; Wey, Blumstein, Shen, \& Jordán, 2008; Whitehead, 2008). and of primates in particular (Brent, Lehmann, \& Ramos-Fernandez, 2011). However, when compared to humans, SNA with primates is often limited by the amount of data that can be gathered on the social relationships of individuals. Traditionally, primate social networks have been studied through standard observation techniques such as scan sampling or focal follows (Altmann, 1974). but these methods are time consuming and provide irregular and sometime biased information (e.g., when one individual is more easily seen or recognized than another; Whitehead, 2008). More recently, the development of GPS collars has provided new ways to gather relatively large amounts of data over substantial periods of time (e.g., Patzelt et al., 2014; Qi et al., 2014). However, GPS techniques have a relatively poor spatial resolution and can only be used to track the movements of groups of individuals (between-group SNA), but not the proximity of individuals within groups. In Patzelt et al. (2014). for instance, two individuals wearing GPS collar are considered associated when they are less than $100 \mathrm{~m}$ away. In this article, we describe a new method to study the sociality of nonhuman primate species on the basis of the automatic collection of proximity data during Automated Learning Device for Monkeys (ALDM; Fagot \& Bonté, 2010; 
Fagot \& Paleressompoulle, 2009) testing (a "proximity network," for short). This new method complements the existing techniques of automatic collection of proximity data that can be used to collect large amounts of data over long periods of times for individuals within groups (within-group SNA).

This method is based on an automatic reinforcement system that has been developed in our laboratory (ALDM test systems). With this system, a group of baboons have free access to computerized testing booths that are installed in trailers next to their enclosure. The baboons are automatically detected and recognized by the computer and are trained to perform cognitive experiments on touchscreens by using positive reinforcement (see the Method section for more details). Baboons can therefore select the testing booth of their choice and maintain visual contact with other individuals taking part in the experiment (through the transparent side walls of the testing booths; see Fig. 1). Earlier studies have shown that this system is an efficient tool for the assessment of cognitive functions in experimental tasks (e.g., memory: Fagot \& De Lillo, 2011; reasoning: Flemming, Thompson, \& Fagot, 2013; or perception: Parron \& Fagot, 2007) and has a positive impact on animal welfare (Fagot, Gullstrand, Kemp, Defilles, \& Mekaouche, 2014).

In the present study, we used standard behavioral observation techniques to establish a social network based on affiliative interactions (interaction network), and compared it to the proximity network based on participation in the ALDM computerized testing. The goals were to determine (1) whether ALDM test systems could serve to inform researchers on the social network of the subjects, and (2) the optimal conditions for the correlation between the proximity and interaction networks to be strongest. To achieve these two goals, we started by establishing a baseline correlation between the proximity network and the interaction network. We then studied the effects of several variables that could affect the correlation to the proximity network. Finally, we also demonstrated that the associations that we observed in the ALDM system truly reflected the social relationships of baboons and could not be explained by other confounding variables.

\section{Material and method}

\section{Pilot study}

The present study was first motivated by a pilot study conducted between 2nd May and 17th May, 2013, on 12 baboons, using the same protocol and analyses described below. This first study showed a strong and reliable positive correlation between the interaction network and the proximity network (Mantel test, $N=12, r=.84,95 \% \mathrm{CI}=[.71 ; .89]$ ), encouraging us to explore this possibility further with a more comprehensive study (see Supplementary Fig. S1). The present study was also motivated by the fact that previous results
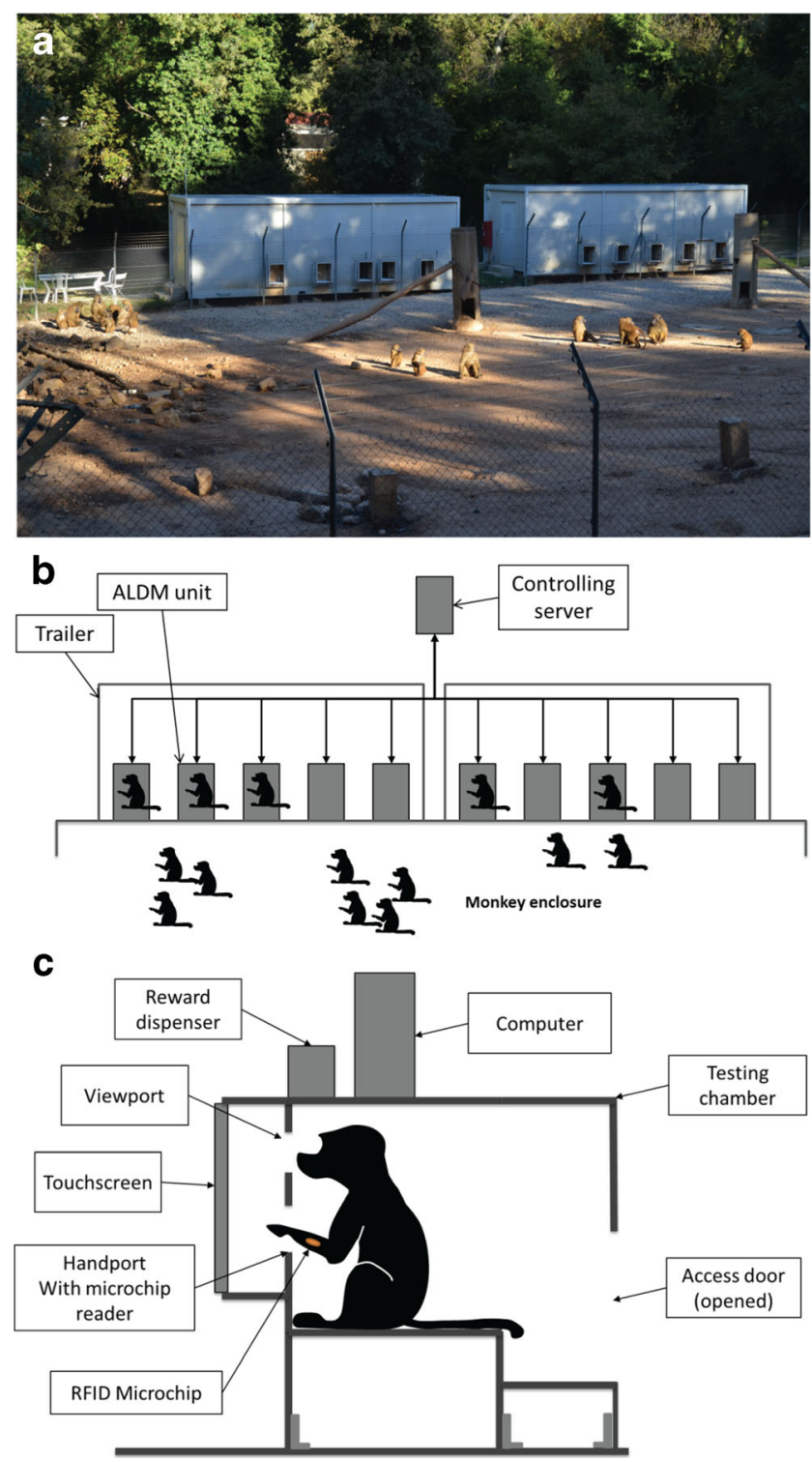

Fig. 1 ALDM self-testing system. (A) Picture of the enclosure, showing the social group of baboons as well as the two trailers with their ten ALDM testing units. (B) Schematic of the ALDM system's organization. (C) Details of the ALDM testing unit

had shown that baboons who can freely participate in cognitive testing tend to be influenced by the presence of others (Huguet, Barbet, Belletier, Monteil, \& Fagot, 2014). suggesting that they form nonrandom associations when they participate in cognitive tests.

\section{Participants}

Twenty-two Guinea baboons (Papio papio) belonging to a large social group of the CNRS Primate Center in Roussetsur-Arc (France) participated in this study. They were seven males (mean age 62 months, $S D=33$ ) and 15 females (mean age 124 months, $S D=75$ ), ranging from 24 to 226 months 
old. The baboons were all marked by two biocompatible $1.2 \times$ $0.2 \mathrm{~cm}$ RFID microchips injected into each forearm.

\section{Ethics statement}

The baboons lived in an outdoor enclosure $\left(700 \mathrm{~m}^{2}\right)$ connected to an indoor area that provided shelter when necessary. The outside enclosure was connected to ten testing booths that the animals could use freely at any time to participate in ALDM testing. This procedure was aimed at preventing the adverse effects that capture and social isolation may entail. The voluntary participation of the subjects reduced stress levels, as inferred from the significant decrease in salivary cortisol levels, as well as the frequency of stereotypies and the amount of inactivity (Fagot et al., 2014). The baboons were neither water- nor food-deprived. Water was provided ad libitum within the enclosure, and the monkeys received their normal ratio of food (fruits, vegetables, and monkey chow) every day at $5 \mathrm{pm}$. The baboons were all born within the primate center.

This research was carried out in accordance with French standards and received approval from the national French ethics committee, the "Comité d'Ethique CE-14 pour l'Expérimentation Animale." The procedures were also consistent with the guidelines of the Association for the Study of Animal Behaviour.

\section{ALDM self-testing procedure}

The study was conducted in a testing facility developed by the last author (Fagot \& Bonté, 2010). The key feature of this facility is that baboons have free access to ALDM computerized testing booths that are installed in trailers next to their enclosure (see Fig. 1). They can thus participate in the computerized testing whenever they choose, and do not need to be captured. The baboons lived inside a $25 \times 30 \mathrm{~m}$ wire-meshed enclosure containing climbing structures for behavioral enrichment. The enclosure is connected to a housing area as well as to ten workstations accessible through holes in the wire mesh. Each ALDM workstation comprises a freely accessible test chamber with transparent side walls that opens to the rear. The front of the test chamber is fitted with a view port $(7 \times$ $7 \mathrm{~cm})$ and two hand ports $(8 \times 5 \mathrm{~cm})$. Looking through the view port allows visual access to a 19-in. LCD touch monitor installed at eye level $25 \mathrm{~cm}$ from the view port. Two antennae are fixed around each arm port, which read the RFID identity number of an animal when one of its forearms is introduced through one of the two arm ports. Identification signals from the microchip are used by the computer to trigger the presentation of the stimulus and to assign behavioral measures (stimulus choices and response times) to each participant. The equipment is controlled by a test program written with EPrime (Psychology Software Tools, Pittsburgh, PA). The test program allows an independent test regimen for each baboon, irrespective of the testing booth used (Fagot \& Paleressompoulle, 2009). Grains of dry wheat are used as rewards (more details can be found in Fagot \& Bonté, 2010; Fagot, Marzouki, Huguet, Gullstrand, \& Claidière, 2015; and Fagot \& Paleressompoulle, 2009). The monkeys could see their partners working in the adjacent workstations of each trailer, but were unable to see their motor responses on the screen: Observational learning was thus impossible.

\section{Computer-based tasks}

The computerized task proposed to the baboons during the behavioral observations was primarily aimed at testing memory processes. Each trial began with a first demonstration slide $(150 \mathrm{~ms})$ containing three $(400 \times 400$ pixels $)$ colored squares on a black background. These squares were randomly located in the center of three of four possible cells, corresponding to the four quarters of the screen. Two of these squares had the same color, and the third was in a different color. After the demonstration slide, the screen turned black for either 0,400 , or $800 \mathrm{~ms}$ (randomly counterbalanced within a block). Then, four $400 \times 400$ gray squares appeared in the response slide, one square within each quarter of the screen. To receive a reward, the baboon had to touch the two gray squares located where the two squares of the same color had appeared in the demonstration slide. The computer recorded which animal was working and which ALDM unit was used, as well as the score and response time for each trial.

\section{Behavioral observations in the enclosure}

The behavioral observations were recorded between the 1 st and 29th of July, 2014. In order to maximize the amount of behavioral information on each of the 22 baboons of the group, we chose to use the scan-sampling method (Altmann, 1974). To carry out the observations, we divided the group into two subgroups of 11 baboons with approximately balanced numbers of individuals per age-sex classes (for details, see Supplementary Table S1). We then recorded two different MP3 soundtracks to control the observation timing. Each soundtrack was composed of a repeated sequence of 11 baboon names whose order was initially randomized and then fixed for every sequence. Each sequence lasted 2 mins, with the names presented every $10 \mathrm{~s}$ and with an additional $10 \mathrm{~s}$ break at the end. Two trained observers recorded the behavior of the group simultaneously for $2 \mathrm{~h}$, with each observer listening to one soundtrack and recording the behavior of the focal baboon when it heard its name.

A total of four observers (two teams of two) alternated with each other every $2 \mathrm{~h}$, from 9 am to $4 \mathrm{pm}$, with sometimes a 1-h overlap used to measure interobserver reliability (see the Procedure section below). Every day, each observer coded the behavior of both baboon subgroups, in order to maintain recognition of the 22 different baboons composing the groups. 
The order of the two teams of observers was also balanced, so that each observer covered every time slot over two days. Observations started at exactly 9:00:00 with stopwatches synchronized to the ALDM system, to precisely determine the time of every behavioral record and to be able to link that behavior to events in the ALDM system.

Height categories of behavior were recorded ("Locomotion," "Object-directed," "Sexual," "Resting," "Self," "Social agonistic," "Social affiliative," and "Other"). The social behaviors were of particular interest to this study and consisted of the following behaviors: "Groom," "Present," "Embrace," "Play, " "Touch," and "Lip-smack" (the full list of behaviors recorded, with their descriptions, can be found in Supplementary Table S2). Monkeys had permanent access to the ALDM workstations during the behavioral observations; when the baboons were in the ALDM system, they could not be observed, and therefore were recorded as "Invisible." Using this technique, we recorded 210 behavioral observations per monkey per day during the study period, corresponding to a total of 79,380 observations for the 22 individuals.

Interobserver reliability was measured during the first and last weeks of the study, totaling $9 \mathrm{~h}$ of simultaneous observations per subgroup of 11 individuals (a total of $18 \mathrm{~h}$ and 5,940 observations). Each possible observer pair had at least $1 \mathrm{~h}$ of simultaneous observations for each subgroup. Cohen's kappa was above $80 \%$ for every possible pair of observers (see Supplementary Table S3), showing that the procedure for observations provided stable and reliable measures of the behaviors.

\section{Statistical analysis}

In order to determine whether a network based on the ALDM system could reflect the social relationships of the baboons, we built two networks: the proximity network, based on spatial proximity during the use of the ALDM system, and the interaction network, based on behavioral observations within the enclosure.

Proximity network To construct the proximity network, we divided the time into periods of $5 \mathrm{~s}$ and calculated, for every possible pair of individuals (e.g., Baboons 1 and 2), the number of periods in which each individual was recorded (i.e., performed a trial; $N_{1}$ for Individual 1 and $N_{2}$ for Individual $2)$, the number of periods in which both individuals were recorded $\left(N_{1-2}\right)$, and the number of periods in which they were both recorded at the same time and in proximity $\left(A_{12}\right)$. As a first step, we defined proximity by the presence at the same time of two individuals in the same trailer (as in Huguet et al., 2014). therefore, up to three ALDM units could separate two individuals considered to be in association (the effect of limiting the measure of association to individuals that were closer is studied below). The simple association index (Whitehead, 2008) was computed as follows: $\alpha_{12}=A_{12} /\left(N_{1}+N_{2}-N_{1-2}\right)$. The association index therefore represents the number of times two individuals were seen in association, divided by the number of times they could have been seen associated.

Interaction network Computation of the interactions network followed the same logic as for the proximity network. We calculated the number of observations in which each individual was recorded (i.e., was observed; $N_{1}$ for Individual 1 and $N_{2}$ for Individual 2), the number of observations in which both individuals were recorded at the same time $\left(N_{12}\right)$, and the number of observations in which they were recorded as interacting $\left(A_{12}\right)$. We calculated two different association

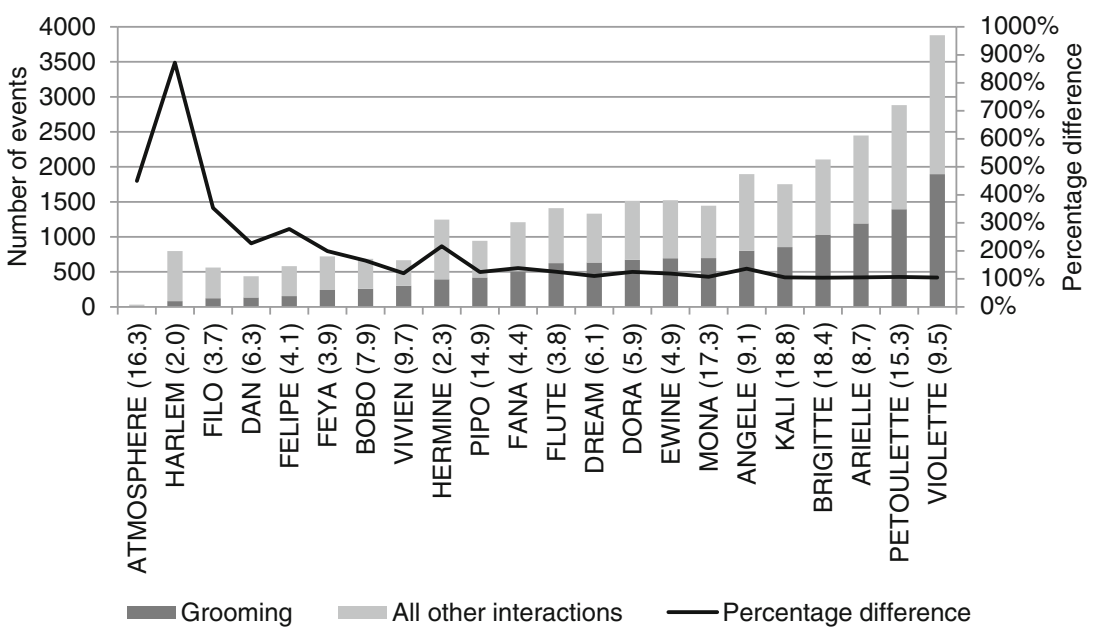

Fig. 2 Distribution of grooming and all other interaction observations per individual (in parentheses is each baboon's age, in years), ordered by the number of grooming events observed (from smallest to largest). The left $y$-axis and bar graph indicate the numbers of grooming events (dark gray) and all other interaction events (light gray), recorded for all individuals in the group (names on the $x$-axis). The right $y$-axis and black line represent the percentage increase in the number of events recorded between grooming events and all interaction events $(200 \%$ means that the number of all interactions events is twice the number of grooming events) 
indexes as above, one for all positive interactions ( $\alpha$-interactions $\left._{12}\right)$ and one limited to grooming only $\left(\alpha\right.$-grooming $\left.{ }_{12}\right)$.

Since network data are nonindependent, we used nonparametric Mantel correlation tests $(p<.05)$ to estimate the correlations between the proximity and interaction matrices (Goslee \& Urban, 2007). and exact permutation tests to create a null distribution of a random choice of testing booth (the number of permutations was set to 10 , 000 in all cases; for a general introduction to network statistics, see Whitehead, 2008). All the analyses and simulations were carried out using R (R Development Core Team, 2015).

\section{Results}

\section{Grooming versus all interactions}

We first computed a grooming network. Since grooming is known to be a major bonding activity between individuals, we expected the grooming network to represent the "true" affiliative relationships between individuals. However, grooming represents a large part of affiliative behaviors for well-connected individuals, but grooming events are less frequent for poorly connected individuals (Fig. 2). Accordingly, we also calculated an interaction network that included grooming as well as all other positive interactions between individuals. As expected, the two networks were highly correlated (Mantel test, $r=.93,95 \% \mathrm{CI}=[.89 ; .97])$.

The two networks were then compared to the proximity network (Fig. 3). The correlation between the proximity network and the grooming network was lower (Mantel test, $r=.35[.24 ; .44])$ than the correlation with all affiliative interactions (Mantel test, $r=.48[.40 ; .53]$ ). The larger correlation found for the all-interaction network can be explained by the fact that some individuals tend to engage in other social activities than grooming (Fig. 2). Using the allinteraction network, therefore, provides a better resolution of the relationships of individuals who are not well integrated into the grooming network. In the remainder of this study, attention was consequently focused on this network made from all affiliative interactions.

\section{Time window to create the proximity network}

Social networks can be very sensitive to the amount of data used in their construction. Whitehead (2008). for instance, gives a rough guideline of a minimum of 30 days of records to construct an accurate network (i.e., a better description of the actual relationships of the individuals). To study the sensitivity of the proximity network to the amount of data used in its
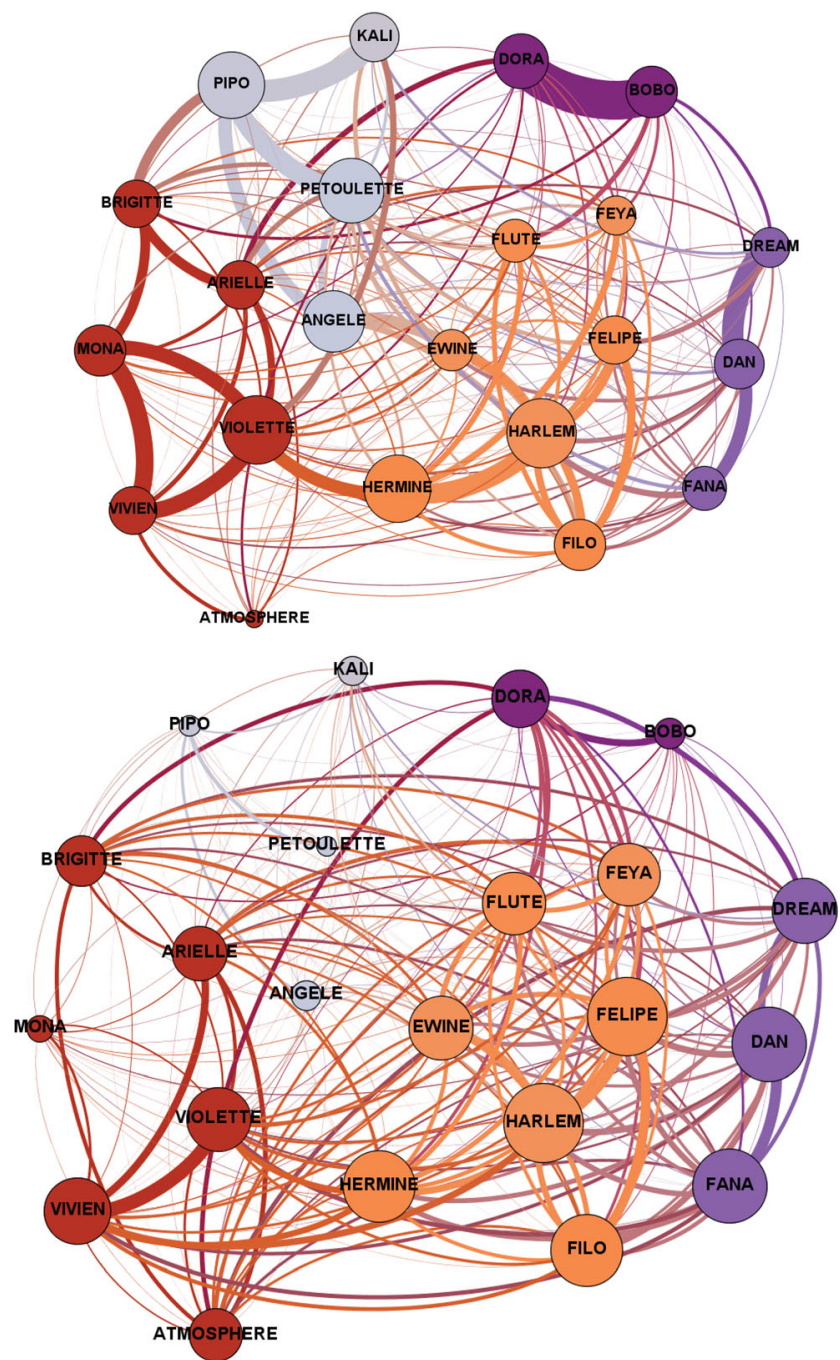

Fig. 3 Visual representation of the interaction (top) and proximity (bottom) networks. The size of the nodes represents the eigenvector centrality of each individual (i.e., how well an individual is connected within the network), and the widths of the links, the strengths of their relationships (their association coefficient, as defined above). The colors are based on modularity classes (i.e., nodes with the same color have dense connections between them and sparse connection with the nodes of other colors), and the layout uses a force-based algorithm (i.e., the distance between individuals is linked to the strength of their relationship; we used the Gephi software [Bastian, Heymann, \& Jacomy, 2009] to represent the networks). The positions of the nodes and their modularity classes (colors) were determined on the basis of the interaction network because it is based on observed interactions between individuals. This structure was kept constant for the proximity network to facilitate comparison (therefore, only the sizes of the links and nodes vary between the two networks; Supplementary Fig. S2 compares the two networks when modularity and position were recalculated for the two networks independently)

construction, we plotted the evolution of the correlation between the proximity and interaction networks as a function of the number of days of data used to determine the proximity network (Fig. 4). Note that in order 


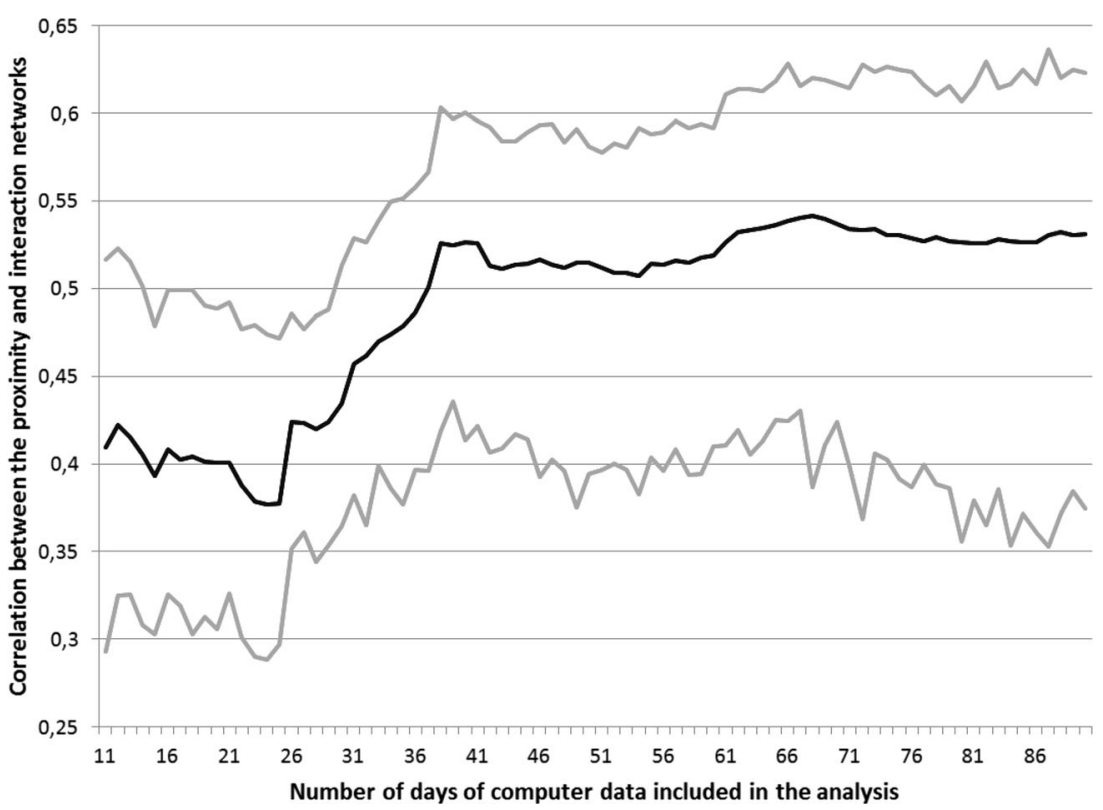

Fig. 4 Correlation between the interaction and proximity networks as a function of the amount of data used to create the proximity network. The $y$-axis shows the correlation (black line) and $95 \%$ CI (gray lines) estimated with a Mantel permutation test (10,000 permutations) for every day of ALDM testing included in the analysis. The $x$-axis shows

to study the effect of the amount of data, we had to include data on a time window (90 days) that is larger than the one used to record the behavioral observations (29 days).

Figure 4 shows that the correlation increased up to about 40 days, and then remained roughly stable. The CIs, however, increased in magnitude after 65 days, probably because the ALDM data used were too far away in time from the interaction network. We therefore chose to use 50 days as an optimal time window in the computation and analyses of the proximity networks. the number of days of data used in the construction of the proximity network (Day 1 is the last day of behavioral observation (29th July), Day 29 is the first day of behavioral observation (1st July), and days with larger numbers are days of computer data that were recorded before the behavioral observations started)

\section{Including individuals who participate more}

Figure 5 represents the extent to which each baboon used the ALDM system during the study period. The average frequencies of daily trials varied greatly between individuals (from 68 to 2,883 tests a day, on average). Since the preceding analysis revealed that a certain amount of data is needed to represent the social bonds precisely, the next analyses explored whether the proximity network provided an accurate reflection of the social relationships only for the baboons that participated most.

Fig. 5 Average numbers of ALDM trials per day per baboon. Error bars represent $S D$ s, and values are calculated based on 50 days

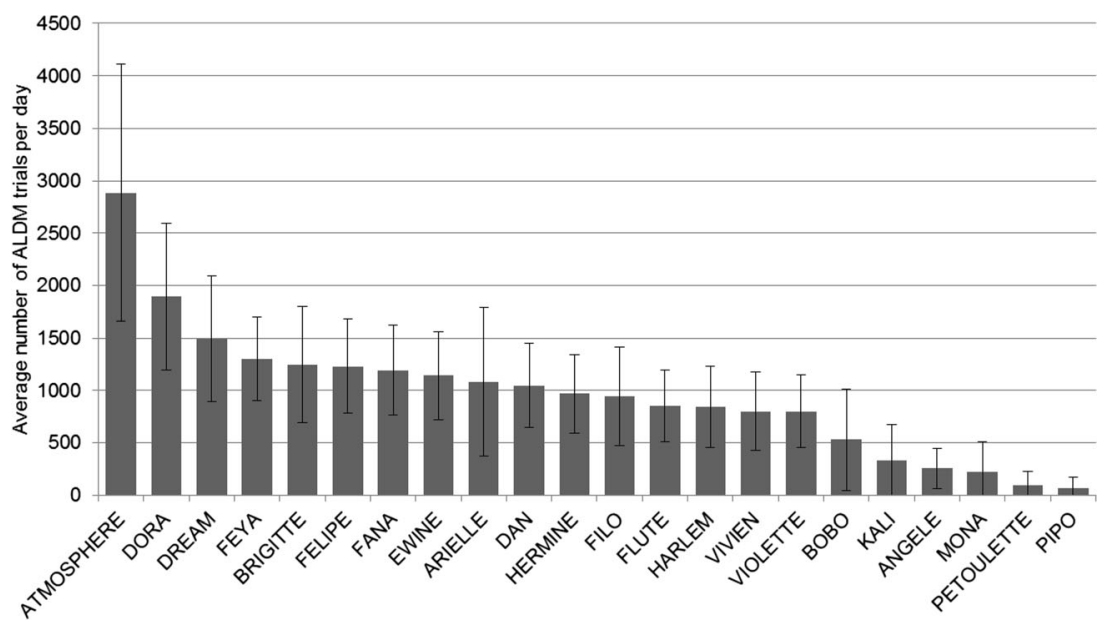




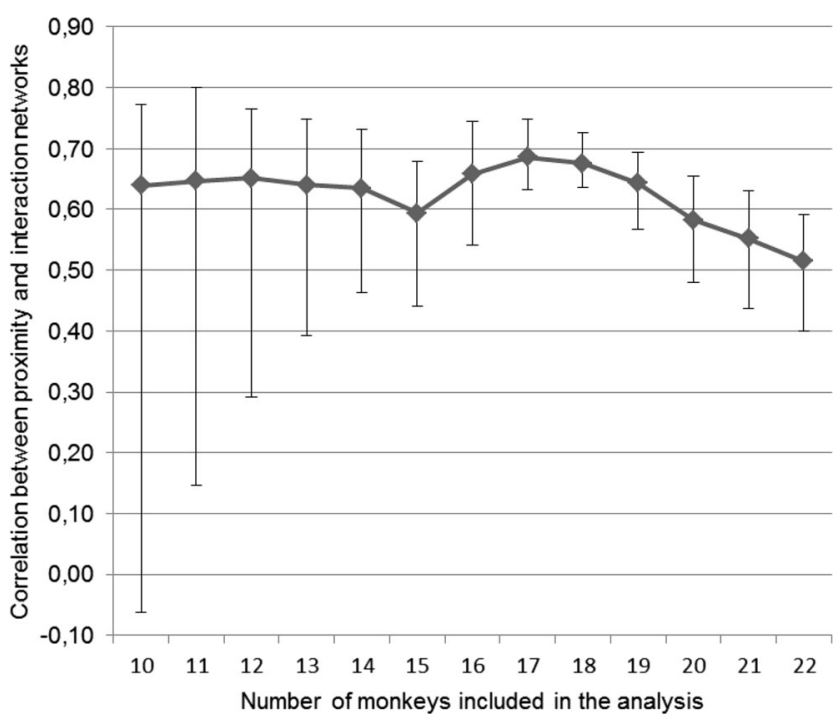

Fig. 6 Correlations between the proximity and interaction networks for baboons with the largest numbers of ALDM trials. On the $x$-axis, "10," for instance, represents the correlation and $95 \%$ confidence interval (error bars) for the two networks including the ten most active individuals in the ALDM test system (from ATMOSPHERE to DAN; see Fig. 5)

To explore the relationship between the number of ALDM trials per monkey and the correlation to the proximity network, we calculated the correlation between the interaction and proximity networks when including only the baboons with the largest number of trials (Fig. 6).

Figure 6 indicates that the estimated correlation remained relatively stable up to 17 individuals, but the precision in the estimate increased greatly with the number of individuals included in the analysis (reduced error bars; note that removing ATMOSPHERE, who participated in ALDM testing a lot, from the 17 individuals did not change the correlation between the two networks significantly: $r=.69[.64 ; .75])$. The decrease observed between 18 and 22 individuals (Kali to Pipo in Fig. 5) corresponds to the decrease in correlation predicted by the low numbers of observations for these baboons. The highest correlation was therefore obtained for 17 individuals, which corresponds to a minimum of 500 measurements per day, on average (and a correlation of $.69[.63 ; .75]$ ). In the remaining analyses, we used these 17 individuals only.

As a sensitivity analysis, we also calculated the correlation obtained after removing the individuals that participated most, and found that the correlation was much lower (Fig. 7). This confirms that the number of ALDM trials per individual, and not the number of individuals, affects the correlation between the social networks.

\section{Effect of the proximity measure}

In the preceding sections, individuals were considered to be associated on the basis of their co-presence in a trailer (as in Huguet et al., 2014). Since five ALDM test systems were installed within each trailer, this initial measure only provided a crude measurement of spatial proximity. In fact, with this initial measure of association, up to three ALDM test systems could separate two individuals. One might expect a more accurate depiction of the interaction network with a finergrained measure of proximity. However, increasing spatial constraints in the measurement of proximity also reduces the number of observed associations, and therefore the correlation. To more closely study the effect of spatial proximity, we calculated the correlations between the interaction and proximity networks when different criteria were used to compute spatial proximity. More precisely, two individuals were considered to be associated when they used adjacent ALDM test units (distance of one), or when their distance was equal to or less than two, three, or four other test systems apart in the trailer (of five units), or for any distance (including test units from both trailers).

Figure 8 indicates that using more stringent criteria to compute spatial proximity within the ALDM test systems decreases the precision (increased error bars, presumably due to a smaller data set) without increasing the correlation. However, there is little difference between the conditions as long as not all ALDM units in the two trailers are considered together. In the remaining of the article, we will focus on the optimal distance of three ALDM units $(r=.70[.65 ; .75])$.

\section{Random association simulations}

The correlation that we observed between the interaction and proximity networks could arise from the preferred association, during computerized experiments, of pairs of baboons that

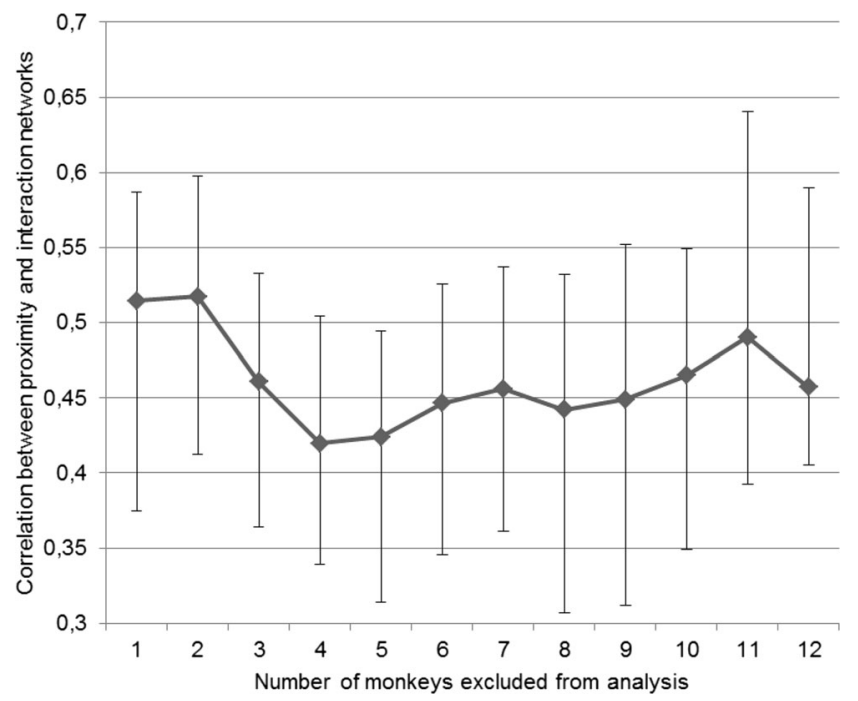

Fig. 7 Correlations for baboons with the smallest numbers of ALDM trials. On the $x$-axis, " 10 ," for instance, represents the correlation found for the networks excluding the ten most frequent individuals (i.e., including the remaining 12 less frequent individuals) 


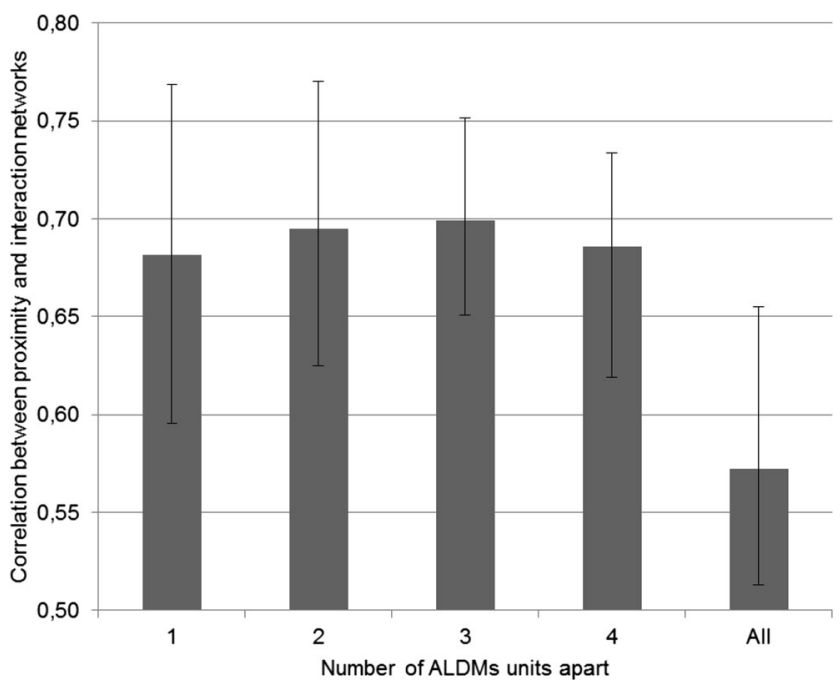

Fig. 8 Correlations between the proximity and interaction networks for different measures of proximity during ALDM testing. On the $x$-axis, the distance between ALDM units is used to define the association between monkeys. Distances 1 to 4 are between two ALDM units in the same trailer, and Distance 4 therefore means that any two baboons using ALDM units in the same trailer are considered to be associated. Distance All means that any two baboons using ALDM units at the same time anywhere, even in two different trailers, are considered associated

have strong social links. According to this hypothesis, pairs that exchange affiliative behaviors frequently tend to preferentially choose to take part in ALDM testing at the same time and in neighboring testing units. An alternative hypothesis, however, is that the correspondence between the two networks arises from factors that are not related to social bonds between individuals. For instance, if certain baboons participate more than others, they might be more likely to be associated, especially if the use of the ten different ALDM units is not uniform (e.g., if certain units are used preferentially at certain times of day). These patterns of behaviors could be independent of the baboons' social relationships and could create nonrandom associations in the proximity network that would lead us to overestimate the importance of social relationships in structuring the proximity network. We used random simulations to firmly establish the role of social relationships as the source of the correlation and definitively rule out possible confounding variables.

To take into account the associations that might arise from nonsocial factors, we used a simulation that randomly selected a baboon for every computer record on the basis of the baboon's frequency of participation in the entire data set. Importantly, only the names of the baboons changed in the data set: the time, the testing unit, and the overall frequency of each baboon in the entire data set were preserved. Once generated, this random data set served to calculate the correlation between this new random proximity network and the interaction network. Consequently, we expected the correlation between the random network and the interaction network to be low if the social relationships of the baboons are responsible for their co-presence in the ALDM units and high if other factors are responsible for their co-presence. We ran a total of 1,000 simulations to obtain the distribution of simulated correlation coefficients and found that the correlation coefficient was equal to -.16 on average (5th-95th percentiles $[-.15 ;-.17])$.

This negative correlation coefficient is explained by the distribution of trials between pairs of individuals in the interaction network. In the random simulation, individuals were drawn at random with a weight equal to their frequency of participation in ALDM testing; individuals that participated often therefore had strong bonds in the resulting network (the correlation between the total number of trials performed by a dyad and their association in the simulated network was
Fig. 9 Distribution of randomly generated correlation coefficients. The $x$-axis shows the value of the correlation between the randomly generated network and the observed interaction network. The $y$-axis shows the number of simulations giving rise to a certain correlation (out of a total of $1,000)$. The observed correlation $(r=.70)$ is indicated by the arrow

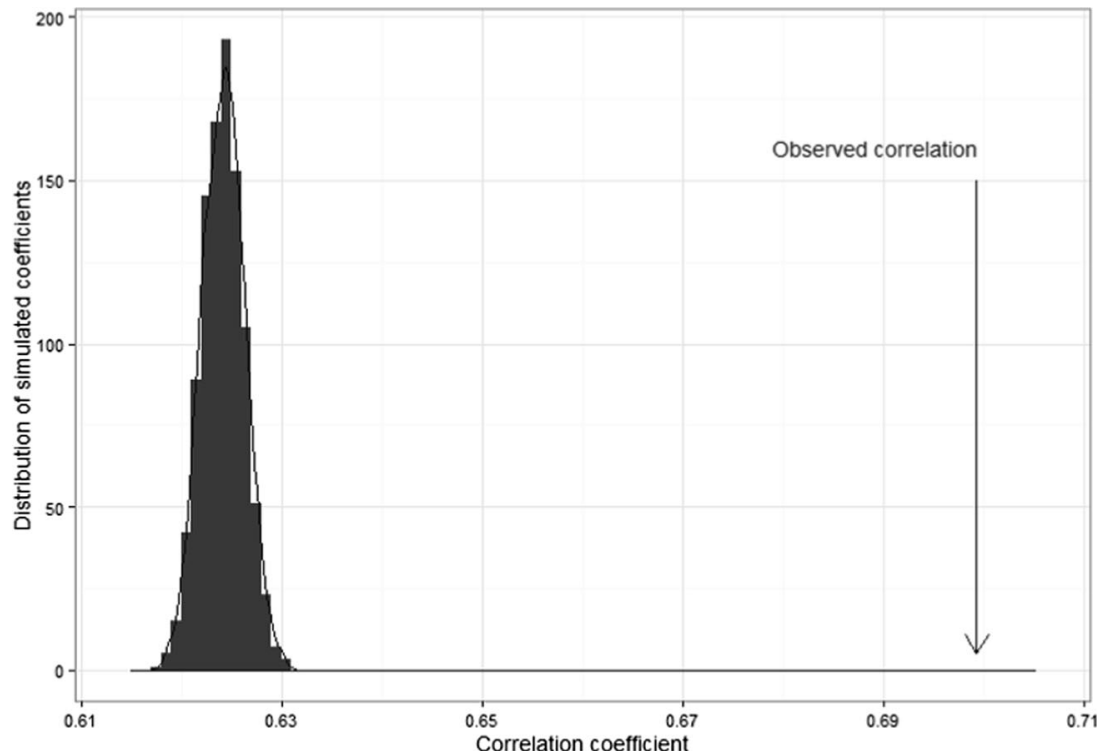


$\left.r_{\mathrm{s}}=.93, p<.001\right)$. On the other hand, we found a small negative correlation between the total number of trials performed by a dyad and their association in the interaction network $\left(r_{\mathrm{s}}=-.16, p=.068\right)$. In other words, baboons that have strong social bonds have a small tendency, on average, to participate less in ALDM testing than baboons with weaker bonds.

This pattern of results can explain, at least partly, why the correlation between the two networks is sensitive to the amount of data used. Behavioral observations will first detect strong relationships, and take more time to detect weak relationships. The opposite happens, to some extent, for the proximity data: Individuals with weak bonds can be detected more easily, on average, because they often take part in ALDM testing, whereas individuals with stronger bonds will require more effort. These results further confirm the validity of our approach and the strength of our results: The correlation obtained between the interaction and proximity networks $(r=.70$ $[.65 ; .75])$ is a consequence of the preferential assortment of the baboons in the ALDM test system, based on their social relationships.

In practice, the social relationships of baboons can affect their participation in the ALDM system in two different and nonexclusive ways. First, baboons that are socially close might participate in ALDM testing simultaneously, but without preferentially choosing adjacent ALDM units. In this case, the synchronous activity of closely linked individuals is what explains our results. Another possibility is that baboons that are socially close preferentially choose ALDM systems that are physically close to each other. In that case, the correspondence between the proximity and interaction networks go beyond a simple foraging synchrony to show a preferential choice of ALDM systems that maintain close physical proximity.

To tease apart the contributions of these two processes, we created a new random simulation in which we randomly permutated the positions of the individuals recorded at the same time. For instance, if baboons A, B, and C were present at the same time in ALDM Units 1, 2, and 3, respectively, the random permutation of their positions could give the result $\mathrm{A}-$ B-C, A-C-B, B-A-C, and so forth, in ALDM Units 1, 2, and 3 , respectively. Therefore, any difference between the randomly generated correlation and the observed one could only be explained by the preferential assortment of closely linked baboons. Using a total of 1,000 simulations, we found that the distribution of randomly generated correlation coefficients was equal to .62 , on average (5th-95th percentiles $[.62 ; .63]$ ) and was significantly less than the observed one (exact permutation test, $N=1,000$ permutations, $p<.001$; see Fig. 9).

\section{Optimal interaction and proximity networks}

The analysis presented above highlights the context in which the proximity network best reflected the interaction network:
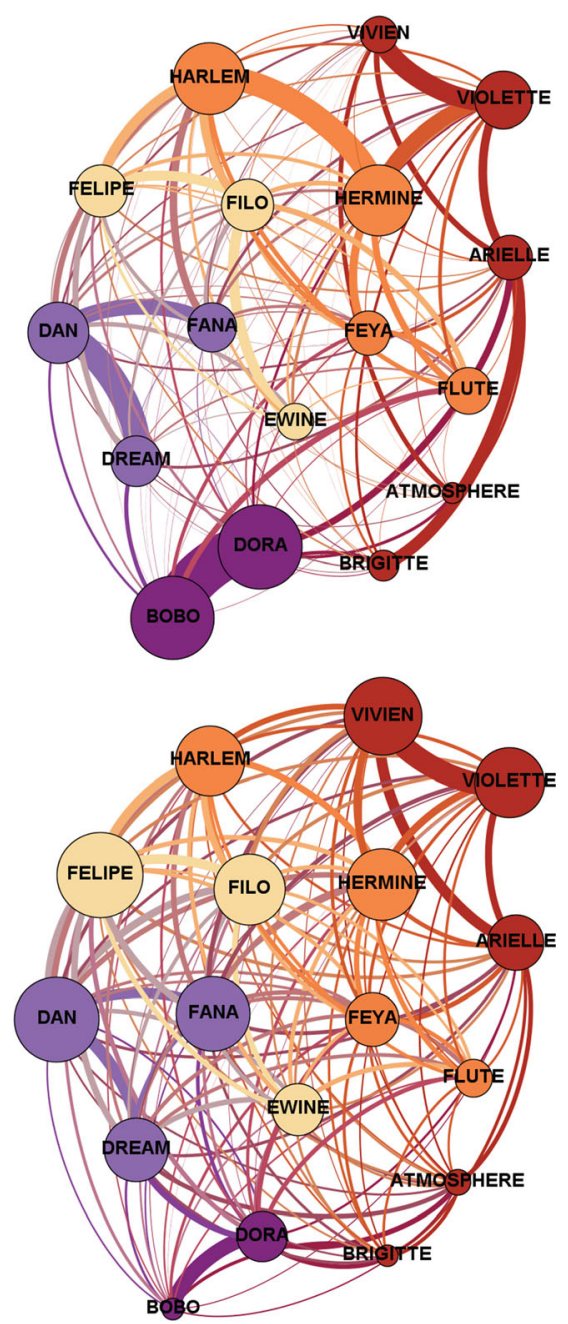

Fig. 10 Visual representation of the interaction (top) and proximity (bottom) networks for the optimal parameters found in the study. The conventions are as in Fig. 3

with 50 days of ALDM testing data from individuals that performed more than 500 ALDM trials on average per day and at a distance of three ALDM units. Inspection of these networks (Fig. 10) shows a high level of similarity.

\section{Discussion}

The aim of the study was to examine the possible use of freely accessible ALDM testing units to describe the social network of a group of baboons. In order to assess the extent to which this proximity-based network reflects the social relationships of individuals, we simultaneously recorded the behavioral interactions of baboons in the enclosure and their participation in cognitive tests, and computed the interaction and proximity networks from these two measures of behavior.

The different statistical analyses conducted above validate the use of the ALDM test systems in social network studies, 
and indicate the context in which the ALDM proximity networks provide the most reliable estimate of the interaction network. The most important factor influencing the correlation between the two networks is the amount of data used to calculate the proximity network. If the data set is too small, the correlation is poor and the confidence interval is large. If the data set is too large, the correlation can be high but the estimate loses precision (large confidence interval), probably because the social network changes over the time: A network based on a broad time window could therefore reflect a sort of average of different stages of this changing network. A number of days between 40 and 60 seems to be optimal, with a relatively constant coefficient and a relatively precise estimate.

The second most important factor is the number of individuals considered in the network. When few individuals are considered, large correlation might arise, but with very low precision (large error bars). If all individuals are considered, on the other hand, the correlation is reduced by the few individuals who do not participate much in cognitive testing (less than 500 trials a day, on average). Not considering the data from the least active ( $n=4-5)$ individuals greatly improves the correlation between the two networks and the precision of the estimates (reduced error bars). Other factors, such as the definition of the proximity used to define association, do not affect the correlation very much.

Third, we found that a network based on all affiliative interactions correlated more with the proximity network than did a network based on grooming only. The network based on all affiliative interactions (including but not limited to grooming) seems to be more representative of the social relationships of the entire group. This might be explained by the fact that most of the social interactions of youngsters take place through play rather than grooming (as is shown in Fig. 2).

Finally, using random simulations, our study demonstrated that the proximity network clearly reflects the social relationships of individuals, rather than other possible, nonsocial constraints: Closely bonded pairs tend to forage in synchrony and to maintain physical proximity during cognitive testing by using neighboring ALDM units.

ALDM testing therefore presents an interesting complementary tool for the study of animal social networks. Like any tool, however, it has certain limitations. For instance, we have shown that the reconstruction of the social network is sensitive to the number of ALDM trials done by each monkey on a daily basis. Attractive experiments, during which the motivation to participate is high, are therefore more likely to produce an accurate network that corresponds more closely to the relationships of the individuals, than would less motivating experiments with less frequent participation. Also, the network might be affected if different individuals were to participate simultaneously in different experiments with variable levels of attractiveness.

Keeping in mind the above limitations, ALDM testing has a very strong potential to access important aspects of the social life of the species being studied. In this study, we focused on the simultaneous presence of individuals in nearby testing units, but the same approach can be followed to study various aspects of the social relationships of individuals. For instance, the succession of individuals in the same unit could give us information about displacement between individuals, and therefore provide access to dominance relationships. Similarly, using the succession of individuals in nearby units, one might have access to information on leadership (within a pair of individuals, which one leads the other to a new activity?). Finally, the continuous monitoring of the social network can be used for group management purposes. We have remarked informally that rapid changes in an individual's network position often result from a difficult situation-for instance, the individual may either be sick or attacked frequently by other group members.

The recent advances in the study of social networks in medicine, the social sciences, and physics offer new tools that can be used by behavioral scientists to ask new questions regarding the social relationships of animals. However, time and costs limit the potential to record detailed social networks over the long term, because behavioral recording requires skill and takes time. The automatic recording of social information on groups of animals to study their social structure and its dynamic changes through time is a promising technological development in the behavioral sciences. New radio tags recording spatial proximity will likely be perfected and become prominent in the future (Barrat, Cattuto, Tozzi, Vanhems, \& Voirin, 2014; Rutz et al., 2012). Compared to these new techniques, the use of a battery of cognitive testing units offers the unique opportunity to collect simultaneously and automatically both the social relationships of individuals and their cognitive performance. In the future, this should allow us to get unprecedented insight into the relationship between cognitive performance in various tasks (such as memory, inhibition, etc.) and the social relationships of individuals - with, ultimately, a dynamic understanding of the links between the two.

Author note The authors declare no competing interests. N.C., J.F., and J.G. gratefully acknowledge financial support from the ASCE program (Grant No. ANR-13-PDOC-0004) of the Agence Nationale de la Recherche. N.C. also gratefully acknowledges financial support from the LICORNES program (Grant No. ANR-12-CULT-0002) of the Agence Nationale de la Recherche. J.F. gratefully acknowledges financial support from Fédération 3C and the EU EUPRIM-Net-II project (No. RII3-026155). The funders had no role in the study design, data collection and analysis, decision to publish, or preparation of the manuscript. This research was conducted at the Rousset-sur-Arc Primate Center (CNRSUPS846), France. The authors thank its staff for technical support, and Diane Austry, Julien Bourdiol, Lauriane Faraut, Aurélien Frick, and Manon Genet for conducting the behavioral observations. All authors 
contributed to the design of the experiment, discussed the results and their implications, and commented on the manuscript at all stages. J.F. developed the ALDM test systems and programmed the experiment. J.G. supervised the behavioral observations. N.C. and A.L. analyzed the results.

\section{References}

Altmann, J. (1974). Observational study of behavior: Sampling methods. Behaviour, 49, 227-267.

Barrat, A., Cattuto, C., Tozzi, A. E., Vanhems, P., \& Voirin, N. (2014). Measuring contact patterns with wearable sensors: Methods, data characteristics and applications to data-driven simulations of infectious diseases. Clinical Microbiology and Infection, 20, 10-16. doi: 10.1111/1469-0691.12472

Bastian, M., Heymann, S., \& Jacomy, M. (2009). Gephi: an open source software for exploring and manipulating networks. The paper was presented on the 17th May 2009 at the International AAAI Conference on Weblogs and Social Media. San Jose, California, USA.

Brent, L. J., Lehmann, J., \& Ramos-Fernandez, G. (2011). Social network analysis in the study of nonhuman primates: A historical perspective. American Journal of Primatology, 73, 720-730. doi:10.1002/ ajp.20949

Croft, D. P., James, R., \& Krause, J. (2008). Exploring animal social networks. Princeton, NJ: Princeton University Press.

Fagot, J., \& Bonté, E. (2010). Automated testing of cognitive performance in monkeys: Use of a battery of computerized test systems by a troop of semi-free-ranging baboons (Papio papio). Behavior Research Methods, 42, 507-516. doi:10.3758/BRM.42.2.507

Fagot, J., \& De Lillo, C. (2011). A comparative study of working memory: Immediate serial spatial recall in baboons (Papio papio) and humans. Neuropsychologia, 49, 3870-3880. doi:10.1016/j. neuropsychologia.2011.10.003

Fagot, J., Gullstrand, J., Kemp, C., Defilles, C., \& Mekaouche, M. (2014). Effects of freely accessible computerized test systems on the spontaneous behaviors and stress level of Guinea baboons (Papio papio). American Journal of Primatology, 76, 56-64. doi: 10.1002/ajp.22193

Fagot, J., Marzouki, Y., Huguet, P., Gullstrand, J., \& Claidière, N. (2015). Using a network of computerized ALDM test systems for the assessment of social cognition in non-human primates. Journal of Visualized Experiments, 99, 52798. doi:10.3791/52798

Fagot, J., \& Paleressompoulle, D. (2009). Automatic testing of cognitive performance in baboons maintained in social groups. Behavior Research Methods, 41, 396-404. doi:10.3758/BRM.41.2.396
Flemming, T. M., Thompson, R. K. R., \& Fagot, J. (2013). Categorical perception and conceptual judgments by nonhuman primates: The paleological monkey and the analogical ape. Cognitive Science, 24, 363-396. doi:10.1016/S0364-0213(00)00029-X

Goslee, S. C., \& Urban, D. L. (2007). The ecodist package for dissimilarity-based analysis of ecological data. Journal of Statistical Software, 22(7), 1-19. doi:10.18637/jss.v022.107

Huguet, P., Barbet, I., Belletier, C., Monteil, J.-M., \& Fagot, J. (2014). Cognitive control under social influence in baboons. Journal of Experimental Psychology: General, 143, 2067-2073. doi:10.1037/ xge0000026

Krause, J., Lusseau, D., \& James, R. (2009). Animal social networks: An introduction. Behavioral Ecology and Sociobiology, 63, 967-973. doi:10.1007/s00265-009-0747-0

Kurvers, R. H. J. M., Krause, J., Croft, D. P., Wilson, A. D. M., \& Wolf, M. (2014). The evolutionary and ecological consequences of animal social networks: Emerging issues. Trends in Ecology \& Evolution, 29, 326-335. doi:10.1016/j.tree.2014.04.002

Parron, C., \& Fagot, J. (2007). Comparison of grouping abilities in humans (Homo sapiens) and baboons (Papio papio) with the Ebbinghaus illusion. Journal of Comparative Psychology, 121, 405-411. doi:10.1037/0735-7036.121.4.405

Patzelt, A., Kopp, G. H., Ndao, I., Kalbitzer, U., Zinner, D., \& Fischer, J. (2014). Male tolerance and male-male bonds in a multilevel primate society. Proceedings of the National Academy of Sciences, 111, 14740-14745. doi:10.1073/pnas.1405811111

Qi, X.-G., Garber, P. A., Ji, W., Huang, Z.-P., Huang, K., Zhang, P., . . L Li, B.-G. (2014). Satellite telemetry and social modeling offer new insights into the origin of primate multilevel societies. Nature Communications, 5, 5296. doi:10.1038/ncomms6296

R Development Core Team. (2015). R: A language and environment for statistical computing. Vienna, Austria: R Foundation for Statistical Computing. Retrieved from www.R-project.org. Accessed date 15 Oct 2014.

Rutz, C., Burns, Z. T., James, R., Ismar, S. M. H., Burt, J., Otis, B., . . St Clair, J. J. H. (2012). Automated mapping of social networks in wild birds. Current Biology, 22, R669-R671. doi:10.1016/j.cub.2012.06. 037

Wey, T., Blumstein, D. T., Shen, W., \& Jordán, F. (2008). Social network analysis of animal behaviour: A promising tool for the study of sociality. Animal Behaviour, 75, 333-344. doi:10.1016/j.anbehav. 2007.06.020

Whitehead, H. (2008). Analyzing animal societies: Quantitative methods for vertebrate social analysis. Chicago, IL: University of Chicago Press. 